\title{
Tracking and Beamforming for Multiple Simultaneous Speakers with Probabilistic Data Association Filters
}

\author{
Tobias Gehrig, Ulrich Klee, John McDonough, Shajith Ikbal, Matthias Wölfel, Christian Fügen \\ Institut für Theoretische Informatik \\ Universität Karlsruhe \\ Am Fasanengarten 5, D-76131 Karlsruhe, Germany \\ email: \{tgehrig, klee, jmcd, shajith, wolfel, fuegen\}@ira.uka.de
}

\begin{abstract}
In prior work, we developed a speaker tracking system based on an extended Kalman filter using time delays of arrival (TDOAs) as acoustic features. While this system functioned well, its utility was limited to scenarios in which a single speaker was to be tracked. In this work, we remove this restriction by generalizing the IEKF, first to a probabilistic data association filter, which incorporates a clutter model for rejection of spurious acoustic events, and then to a joint probabilistic data association filter (JPDAF), which maintains a separate state vector for each active speaker. In a set of experiments conducted on seminar and meeting data, the JPDAF speaker tracking system reduced the multiple object tracking errror from $20.7 \%$ to $14.3 \%$ with respect to the IEKF system. In a set of automatic speech recognition experiments conducted on the output of a 64 channel microphone array which was beamformed using automatic speaker position estimates, applying the JPDAF tracking system reduced word error rate from $67.3 \%$ to $66.0 \%$. Moreover, the word error rate on the beamformed output was $13.0 \%$ absolute lower than on a single channel of the array.

Index Terms: acoustic source localization, Kalman filter, person tracking, far-field speech recognition, microphone arrays
\end{abstract}

\section{Introduction}

In prior work on acoustic speaker tracking [1], we used an extended Kalman filter to directly update the speaker position estimate based on a set of observed time delays of arrival (TDOAs). In particular, the TDOAs comprised the observation associated with an extended Kalman filter whose state corresponded to the speaker position. Hence, the new position estimate came directly from the update formulae associated with the Kalman filter. We tested our algorithm on seminar data involving actual human subjects, and found that our algorithm provided localization performance superior to the standard techniques such as [2]. In other work [3], we enhanced our audio localizer with video information.

Although the systems described in our prior work functioned well, their utility was limited to scenarios wherein a single subject was to be tracked. In this work, we seek to remove this limitation and develop a system that can track several simultaneous speakers, such as might be required for meeting and small conference scenarios. Our approach is based on two generalizations of the IEKF, namely, the probabilistic data association filter (PDAF) and the joint probabilistic data association filter (JPDAF). Such data association filters have been used extensively in the computer vision field [4], but have seen less widespread use in the field of acoustic person localization and tracking [5]. The JPDAF is able to track multiple, simultaneous speakers, which is not possible with the simple IEKF. As we show here, this capacity for tracking multiple active speakers is the primary reason why the JPDAF system provides tracking performance superior to that achieved with the IEKF.

One of the primary uses of an acoustic person tracking or localization system is for beamforming the multi-channel output of a microphone array to focus on the speech of a given speaker. This beamformed output can be used as the input of a speech-to-text (STT) or automatic speech recognition system. In addition to results illustrating the accuracy of the source localization procedures discussed here, we also report the results of a set of STT experiments conducted on the beamformed output of a 64 channel microphone array. The latter set of experiments demonstrates that the improved speaker tracking accuracy of the JPDAF leads to improved accuracy of a far field STT system.

The balance of this work is organized as follows. In Section 2, we review the process of source localization based on time-delay of arrival estimation. In particular, we formulate source localization as a problem in nonlinear least squares estimation, then develop an appropriate linearized model. Section 3 provides a brief exposition of the extended Kalman filter, as well as it variants, the PDAF and JPDAF. Section 4 presents the results of our initial experiments comparing the tracking performance of the IEKF and JPDAF, as well as the performance of far field STT systems based on the two.

\section{Source Localization}

Consider the $i$-th pair of microphones, and let $\mathbf{m}_{i 1}$ and $\mathbf{m}_{i 2}$ respectively be the positions of the first and second microphones in the pair. Let $\mathbf{x}$ denote the position of the speaker in $\mathbf{R}^{3}$. Then the TDOA between the pair of microphones is

$$
T_{i}(\mathbf{x})=T\left(\mathbf{m}_{i 1}, \mathbf{m}_{i 2}, \mathbf{x}\right)=\frac{1}{s}\left(d_{i 1}-d_{i 2}\right)
$$

where $d_{i j}=\left\|\mathbf{x}-\mathbf{m}_{i j}\right\|$ is the distance from the source to microphone $\mathbf{m}_{i j}$ and $s$ is the speed of sound.

Source localization based on a maximum likelihood (ML) criterion [6] proceeds by minimizing the error function

$$
\epsilon(\mathbf{x})=\sum_{i=0}^{N-1} \frac{1}{\sigma_{i}^{2}}\left[\hat{\tau}_{i}-T_{i}(\mathbf{x})\right]^{2}
$$

where $\hat{\tau}_{i}$ is the observed TDOA for the $i$-th microphone pair, $\sigma_{i}^{2}$ is the error covariance associated with this observation, and $N$ is the number of unique microphone pairs. The TDOAs can be estimated 
with a variety of well-known techniques [7, 8]. Perhaps the most popular method involves phase transform (PHAT), a variant of the generalized cross correlation (GCC) which can be expressed as

$$
R_{12}(\tau)=\frac{1}{2 \pi} \int_{-\pi}^{\pi} \frac{X_{1}\left(e^{j \omega \tau}\right) X_{2}^{*}\left(e^{j \omega \tau}\right)}{\left|X_{1}\left(e^{j \omega \tau}\right) X_{2}^{*}\left(e^{j \omega \tau}\right)\right|} e^{j \omega \tau} d \omega
$$

The TDOA estimate is then given by $\hat{\tau}_{i}=\max _{\tau} R_{12}(\tau)$.

In earlier work [1], we showed how the nonlinear least squares criterion (2) can be linearized about the current position estimate. In particular, we defined

$$
\boldsymbol{\Sigma}=\operatorname{diag}\left[\begin{array}{llll}
\sigma_{0}^{2} & \sigma_{1}^{2} & \cdots & \sigma_{N-1}^{2}
\end{array}\right]
$$

and derived the linearized criterion

$$
\epsilon(\mathbf{x} ; t)=[\overline{\boldsymbol{\tau}}(t)-\mathbf{C}(t) \mathbf{x}]^{T} \boldsymbol{\Sigma}^{-1}[\overline{\boldsymbol{\tau}}(t)-\mathbf{C}(t) \mathbf{x}]
$$

where $\overline{\boldsymbol{\tau}}(t)$ and $\mathbf{C}(t)$ are defined in [1].

While (5) is sufficient to estimate the position of a speaker at any given time instant, it takes no account of past observations, which may also be useful for determining the speaker's current position. This can be achieved, however, by defining a model of the speaker's dynamics, and applying an extended Kalman filter to this nonlinear regression problem.

\section{Kalman Filters}

Here we briefly review the extended Kalman filter (EKF) and its variations, the PDAF and JPDAF.

\subsection{Extended Kalman Filter}

Let $\mathbf{x}(t)$ denote the current state of a Kalman filter and $\mathbf{y}(t)$ the current observation. As $\mathbf{x}(t)$ cannot be observed directly, it must be inferred from the time series $\{\mathbf{y}(t)\}_{t}$; this is the primary function of the Kalman filter. The operation of the Kalman filter is governed by a state space model consisting of a process and an observation equation, respectively,

$$
\begin{aligned}
\mathbf{x}(t+1) & =\mathbf{F}(t+1, t) \mathbf{x}(t)+\boldsymbol{\nu}_{1}(t) \\
\mathbf{y}(t) & =\mathbf{C}(t, \mathbf{x}(t))+\boldsymbol{\nu}_{2}(t)
\end{aligned}
$$

where $\mathbf{F}(t+1, t)$ is a known transition matrix. The term $\mathbf{C}(t, \mathbf{x}(t))$ is the known observation functional, which can represent any arbitrary, nonlinear, time varying mapping from $\mathbf{x}(t)$ to $\mathbf{y}(t)$. In (6-7) the process and observation noise terms are denoted by $\boldsymbol{\nu}_{1}(t)$ and $\boldsymbol{\nu}_{2}(t)$ respectively. These noise terms are by assumption zero mean, white Gaussian random vector processes with covariance matrices $\mathbf{Q}_{i}(t)$ for $i=1,2$.

In the sequel, it will prove useful to define two estimates of the current state: Let $\hat{\mathbf{x}}\left(t \mid \mathcal{Y}_{t-1}\right)$ denote the predicted state estimate of $\mathbf{x}(t)$ obtained from all observations $\mathcal{Y}_{t-1}=\{\mathbf{y}(i)\}_{i=0}^{t-1}$ up to time $t-1$. The filtered state estimate $\hat{\mathbf{x}}\left(t \mid \mathcal{Y}_{t}\right)$, on the other hand, is based on all observations $\mathcal{Y}_{t}=\{\mathbf{y}(i)\}_{i=0}^{t}$ including the current one. The predicted observation is then given by

$$
\hat{\mathbf{y}}\left(t \mid \mathcal{Y}_{t-1}\right)=\mathbf{C}\left(t, \hat{\mathbf{x}}\left(t \mid \mathcal{Y}_{t-1}\right)\right)
$$

which follows readily from (7). By definition, the innovation is the difference

$$
\boldsymbol{\alpha}(t)=\mathbf{y}(t)-\hat{\mathbf{y}}\left(t \mid \mathcal{Y}_{t-1}\right)
$$

between actual and predicted observations. Generalizing the classical Kalman filter to the EKF entails linearizing $\mathbf{C}(t, \mathbf{x}(t))$ about the predicted state estimate $\hat{\mathbf{x}}\left(t \mid \mathcal{Y}_{t-1}\right)$. Let us denote this linearization as $\mathbf{C}(t)$.

The correlation matrix $\mathbf{R}(t)=\mathcal{E}\left\{\boldsymbol{\alpha}(t) \boldsymbol{\alpha}^{T}(t)\right\}$ of the innovations sequence can be calculated from $[9, \S 10.3]$

$$
\mathbf{R}(t)=\mathbf{C}(t) \mathbf{K}(t, t-1) \mathbf{C}^{T}(t)+\mathbf{Q}_{2}(t)
$$

where $\mathbf{K}(t, t-1)=\mathcal{E}\left\{\boldsymbol{\epsilon}(t, t-1) \boldsymbol{\epsilon}^{T}(t, t-1)\right\}$ is the correlation matrix of the predicted state error, $\boldsymbol{\epsilon}(t, t-1)=\mathbf{x}(t)-\hat{\mathbf{x}}\left(t \mid \mathcal{Y}_{t-1}\right)$. The Kalman gain for the EKF is defined as $[9, \S 10.10]$

$$
\begin{aligned}
\mathbf{G}_{F}(t) & =\mathbf{F}^{-1}(t+1, t) \mathcal{E}\left\{\mathbf{x}(t+1) \boldsymbol{\alpha}^{T}(t)\right\} \mathbf{R}^{-1}(t) \\
& =\mathbf{K}(t, t-1) \mathbf{C}^{T}(t) \mathbf{R}^{-1}(t)
\end{aligned}
$$

To calculate $\mathbf{G}_{F}(t)$, we must know $\mathbf{K}(t, t-1)$ in advance. The latter is available from the Riccati equation, which can be stated as $[9, \S 10.4]$

$$
\begin{aligned}
\mathbf{K}(t+1, t) & =\mathbf{F}(t+1, t) \mathbf{K}(t) \mathbf{F}^{T}(t+1, t)+\mathbf{Q}_{1}(t) \\
\mathbf{K}(t) & =[\mathbf{I}-\mathbf{F}(t, t+1) \mathbf{G}(t) \mathbf{C}(t)] \mathbf{K}(t, t-1)
\end{aligned}
$$

where $\mathbf{K}(t)=\mathcal{E}\left\{\boldsymbol{\epsilon}(t) \boldsymbol{\epsilon}^{T}(t)\right\}$ is the correlation matrix of the filtered state error, $\boldsymbol{\epsilon}(t)=\mathbf{x}(t)-\hat{\mathbf{x}}\left(t \mid \mathcal{Y}_{t}\right)$.

An update of the state estimate proceeds in two steps: First, the predicted state estimate $\hat{\mathbf{x}}\left(t \mid \mathcal{Y}_{t-1}\right)=\mathbf{F}(t, t-1) \hat{\mathbf{x}}\left(t-1 \mid \mathcal{Y}_{t-1}\right)$ is formed and used to calculate the innovation $\alpha(\mathrm{t})$ as in (8-9), as well as the linearized observation functional $\mathbf{C}(t)$. Then the correction based on the current observation is applied to obtain the filtered state estimate according to $[9, \S 10.4]$

$$
\hat{\mathbf{x}}\left(t \mid \mathcal{Y}_{t}\right)=\hat{\mathbf{x}}\left(t \mid \mathcal{Y}_{t-1}\right)+\mathbf{G}_{F}(t) \boldsymbol{\alpha}(t)
$$

For the sake of simplicity of exposition, we shall base our development on the EKF in the sequel; details of IEKF can be found in [1].

To construct a speaker tracking system, we need only associate the observation $\mathbf{y}(t)$ with the TDOA estimate $\boldsymbol{\tau}(t)$ for the audio features. The observation functional $\mathbf{C}\left(t, \hat{\mathbf{x}}\left(t \mid \mathcal{Y}_{t-1}\right)\right)$ required for the Kalman filter is formulated as a mapping from the speaker position $\mathbf{x}$ to a vector of time delays $\boldsymbol{\tau}(t)$, as in (1). The TDOA error covariance matrix $\boldsymbol{\Sigma}$ in (4) can be associated with the observation noise covariance $\mathbf{Q}_{2}(t)$. Hence, we have all relations needed on the observation side of the Kalman filter. We need only supplement these with an appropriate model of the speaker's dynamics to develop an algorithm capable of tracking a moving speaker, as opposed to finding his position at a single time instant.

Consider the simplest model of speaker dynamics, wherein the speaker is "stationary" inasmuch as he moves only under the influence of the process noise $\boldsymbol{\nu}_{1}(t)$. The transition matrix is then $\mathbf{F}(t+1 \mid t)=\mathbf{I}$. Assuming the process noise components in the three directions are statistically independent, we can set $\mathbf{Q}_{1}(t)=\sigma^{2} T^{2} \mathbf{I}$ where $T$ is the time since the last state update. Note that $T$ can vary given that a speaker does not always speak, and no update is possible when the speaker is silent.

\subsection{Probabilistic Data Association Filter}

The PDAF is a generalization of the Kalman filter wherein the Gaussian probability density function (pdf) associated with the location of the speaker or target is supplemented with a pdf for random false alarms or clutter [10, 66.4$]$. Through the inclusion of the clutter model, the PDAF is able to make use of several observations $\left\{\mathbf{y}_{i}(t)\right\}_{i=1}^{m_{t}}$ for each time instant, where $m_{t}$ is the total 
number of observations for time $t$. Each observation can then be attributed either to the target itself, or to the background model. Let us define the association events

$$
\begin{aligned}
\theta_{i}(t) & =\left\{\mathbf{y}_{i}(t) \text { is the target observation at time } t\right\} \\
\theta_{0}(t) & =\{\text { all observations are clutter }\}
\end{aligned}
$$

and the posterior probability of each event

$$
\beta_{i}(t)=P\left\{\theta_{i}(t) \mid \mathcal{Y}_{t}\right\}
$$

As the events $\left\{\theta_{i}(t)\right\}_{i=0}^{m_{t}}$ are exhaustive and mutually exclusive, we have $\sum_{i=0}^{m_{t}} \beta_{i}(t)=1$. Moreover, invoking the total probability theorem, the filtered state estimate can be expressed as

$$
\hat{\mathbf{x}}\left(t \mid \mathcal{Y}_{t}\right)=\sum_{i=0}^{m_{t}} \hat{\mathbf{x}}_{i}\left(t \mid \mathcal{Y}_{t}\right) \beta_{i}(t)
$$

where $\hat{\mathbf{x}}_{i}\left(t \mid \mathcal{Y}_{t}\right)=E\left\{\mathbf{x}(t) \mid \theta_{i}(t), \mathcal{Y}_{t}\right\}$ is the updated state estimate conditioned on $\theta_{i}(t)$. It can be readily shown that this state estimate can be calculated as

$$
\hat{\mathbf{x}}_{i}\left(t \mid \mathcal{Y}_{t}\right)=\hat{\mathbf{x}}\left(t \mid \mathcal{Y}_{t}\right)+\mathbf{G}_{F}\left(t, \hat{\mathbf{x}}\left(t \mid \mathcal{Y}_{t-1}\right)\right) \boldsymbol{\alpha}_{i}\left(t, \hat{\mathbf{x}}\left(t \mid \mathcal{Y}_{t-1}\right)\right)
$$

where

$$
\boldsymbol{\alpha}_{i}\left(t, \hat{\mathbf{x}}\left(t \mid \mathcal{Y}_{t-1}\right)\right)=\mathbf{y}_{i}(t)-\mathbf{C}\left(t, \hat{\mathbf{x}}\left(t \mid \mathcal{Y}_{t-1}\right)\right)
$$

is the innovation for observation $\mathbf{y}_{i}(t)$. The combined update is

$$
\hat{\mathbf{x}}\left(t \mid \mathcal{Y}_{t}\right)=\hat{\mathbf{x}}\left(t \mid \mathcal{Y}_{t-1}\right)+\mathbf{G}_{F}\left(t, \hat{\mathbf{x}}\left(t \mid \mathcal{Y}_{t-1}\right)\right) \boldsymbol{\alpha}\left(t, \hat{\mathbf{x}}\left(t \mid \mathcal{Y}_{t-1}\right)\right)
$$

where the combined innovation is

$$
\boldsymbol{\alpha}\left(t, \hat{\mathbf{x}}\left(t \mid \mathcal{Y}_{t-1}\right)\right)=\sum_{i=1}^{m_{t}} \boldsymbol{\alpha}_{i}\left(t, \hat{\mathbf{x}}\left(t \mid \mathcal{Y}_{t-1}\right)\right) \beta_{i}(t)
$$

The Riccati equation (11-12) must be suitably modified to account for the additional uncertainty associated with the multiple innovations $\left\{\boldsymbol{\alpha}_{i}(t)\right\}$, as well as the possibility of the null event $\theta_{0}(t)$; see Bar-Shalom and Fortmann $[10, \S 6.4]$ for details.

\subsection{Joint Probabilistic Data Association Filter}

The JPDAF is an extension of the PDAF to the case of multiple targets. Consider the set $\mathbf{Y}(t)=\left\{\mathbf{y}_{i}(t)\right\}_{i=1}^{m_{t}}$ of all observations occuring at time instant $t$ and let $\mathcal{Y}_{t-1}=\{\mathbf{Y}(i)\}_{i=0}^{t-1}$ denote the set of all past observations. The first step in the JPDA algorithm is the evaluation of the conditional probabilities of the joint association events

$$
\boldsymbol{\theta}=\bigcap_{i=1}^{m_{t}} \theta_{i k_{i}}
$$

where the atomic events are defined as

$$
\theta_{i k}=\{\text { observation } i \text { originated from target } k\}
$$

for all $i=1, \ldots, m_{t} ; t=0,1, \ldots, T$. Here, $k_{i}$ denotes the index of the target to which the $i$-th observation is associated in the event currently under consideration. A feasible event is defined as an event wherein

1. An observation has exactly one source, which can be the clutter model;

2. No more than one observation can originate from any target.
In the acoustic person tracking application where the observations are peaks in the cross correlation function for pairs of microphones, the second point must be interpreted as referring to the observations for any given pair of microphones. Applying Bayes' rule, the conditional probability of $\boldsymbol{\theta}(t)$ can be expressed as

$$
P\left\{\boldsymbol{\theta}(t) \mid \mathcal{Y}_{t}\right\}=\frac{P\left\{\mathbf{Y}(t) \mid \boldsymbol{\theta}(t), \mathcal{Y}_{t-1}\right\} P\{\boldsymbol{\theta}(t)\}}{P\left\{\mathbf{Y}(t) \mid \mathcal{Y}_{t-1}\right\}}
$$

where the marginal probability $P\left\{\mathbf{Y}(t) \mid \mathcal{Y}_{t-1}\right\}$ is computed by summing the joint probability in the numerator of (20) over all possible $\boldsymbol{\theta}(t)$. The conditional probability of $\mathbf{Y}(t)$ required in (20) can be calculated from

$$
P\left\{\mathbf{Y}(t) \mid \boldsymbol{\theta}(t), \mathcal{Y}_{t-1}\right\}=\prod_{i=1}^{m_{t}} p\left(\mathbf{y}_{i}(t) \mid \theta_{i k_{i}}(t), \mathcal{Y}_{t-1}\right)
$$

The individual probabilities on the right side of (21) can be easily evaluated given the fundamental assumption of the JPDAF, namely,

$$
\mathbf{y}_{i}(t) \backsim \mathcal{N}\left(\hat{\mathbf{y}}_{k_{i}}\left(t \mid \mathcal{Y}_{t-1}\right), \mathbf{R}_{k_{i}}(t)\right)
$$

where $\hat{\mathbf{y}}_{k_{i}}\left(t \mid \mathcal{Y}_{t-1}\right)$ and $\mathbf{R}_{k_{i}}(t)$ are, respectively, the predicted observation (8) and innovation covariance matrix (10) for target $k_{i}$. The prior probability $P\{\boldsymbol{\theta}(t)\}$ in (20) can be readily evaluated through combinatorial arguments [10, $\S 9.3]$. Once the posterior probabilities of the joint events $\{\boldsymbol{\theta}(t)\}$ have been evaluated for all targets together, the state update for each target can be made separately according to (17-19).

As the JPDAF can track multiple targets, it was necessary to formulate rules for deciding when a new target should be created, when two targets should be merged and when a target should be deleted. Details of the heuristics used for this purpose can be found in [11].

\section{Experiments}

The test set used to evaluate the algorithms proposed here contains approximately three hours of audio and video data recorded during 18 seminars held by students and faculty at University of Karlsruhe (UKA) in Karlsruhe, Germany. An additional hour of test data was recorded at Athens Information Technology in Athens, Greece, IBM at Yorktown Heights, New York, USA, Instituto Trentino di Cultura in Trento, Italy, and Universitat Politecnica de Catalunya in Barcelona, Spain. These recordings were made in connection with the European Union integrated project CHIL, Computers in the Human Interaction Loop. In the sequel, we describe out speaker tracking and STT experiments.

\subsection{Speaker Tracking Experiments}

Prior to the start of the recordings, four video cameras in the corners of the room had been calibrated with the technique of Zhang [12]. The location of the centroid of the speaker's head in the images from the four calibrated video cameras was manually marked every second. Using these hand-marked labels, the true position of the speaker's head in the three dimensions was calculated using the technique described in [12]. These "ground truth" speaker positions are accurate to within $10 \mathrm{~cm}$. For the speaker tracking experiments described here, the seminars were recorded with several four-element T-shaped arrays. A precise description of the sensor and room configuration at UKA is provided in [1].

Tracking performance was evaluated only on those parts of the seminars where only a single speaker was active. For these parts, 


\begin{tabular}{|c|c||c|r|r|c|}
\hline Filter & Test Set & $\begin{array}{c}\text { MOTP } \\
(\mathrm{cm})\end{array}$ & \% Miss & \% FP & \% MOTE \\
\hline IEKF & lecture & 11.4 & 8.32 & 8.30 & 16.6 \\
IEKF & interactive & 18.0 & 28.75 & 28.75 & 57.5 \\
IEKF & complete & 12.1 & 10.37 & 10.35 & 20.7 \\
JPDAF & lecture & 11.6 & 5.81 & 5.78 & 11.6 \\
JPDAF & interactive & 17.7 & 19.60 & 19.60 & 39.2 \\
JPDAF & complete & 12.3 & 7.19 & 7.16 & 14.3 \\
\hline
\end{tabular}

Table 1: Speaker tracking performance for IEKF and JPDAF systems.

it was determined whether the error between the ground truth and the estimated position is less $50 \mathrm{~cm}$. Any instance where the error exceeded this threshold was treated as a false positive (FP) and was not considered when calculating the multiple object tracking precision (MOTP), which is defined as the average horizontal position error. If no estimate fell within $50 \mathrm{~cm}$ of the ground truth, it was treated as a miss. Letting $N_{\mathrm{fp}}$ and $N_{\mathrm{m}}$, respectively, denote the total number of false positives and misses, the multiple object tracking error (MOTE) is defined as $\left(N_{\mathrm{fp}}+N_{\mathrm{m}}\right) / N$ where $N$ is the total number of ground truth positions. We evaluated performance separately for the portion of the seminar during which only the lecturer spoke, and that during which the lecturer interacted with the audience. Shown in Table 4.1 are the results of our experiments. These results clearly show that the JPDAF provided better tracking performance for both the lecture and interactive portions of the seminar. As one might expect, the reduction in MOTE was largest for the interactive portion, where multiple speakers were often simultaneously active.

\subsection{STT Experiments}

For the purpose of beamforming and STT experiments, the seminars were also recorded with a 64 channel Mark III microphone array developed at the US National Institute of Standards and Technologies (NIST); see [1] for the placement of the various sensors. The training procedures and data sets used for training the acoustic and language model components of the STT systems used for the experiments undertaken here are described in Fügen et al [13], as are the automatic speech segmentation and speaker clustering procedures. Our STT experiments were conducted on two sets of data: the first was the development data distributed to all participants in the NIST RT06 speech-to-text evaluation, which consisted of approximately 3.5 hours of CHIL seminar data recorded with the Tarrays as well as the Mark III. The second set was the NIST RT06 evaluation data, which was similar in nature and duration to the development data. Experiments on the evaluation data, however, were "blind" in that no transcriptions were available for system development. Rather, the raw STT hypotheses were submitted to NIST as part of the RT06 evaluation. NIST then scored the hypotheses against undisclosed references, tabulated the scores, and distributed the results to all RT06 participants.

Shown in Table 4.2 are the results of our STT experiments, wherein we compared word error rates (WERs) for a single channel of the Mark III, as well as the beamformed output of the entire array using automatic position estimates based on the IEKF and the JPDAF. Clearly, both beamformed outputs provided word error rates more than $12 \%$ absolute lower than the single channel. Moreover, it is apparent that beamforming based on the position estimates returned by the JPDAF provides significantly better STT performance than beamforming on the IEKF position estimates.

\begin{tabular}{|c||c|c|c|}
\hline \multicolumn{1}{|c||}{} & \multicolumn{4}{c|}{$\%$ Word Error Rate } \\
Test Set & Single Channel & IEKF & JPDAF \\
\hline RT06 Dev & 61.8 & 49.4 & 48.8 \\
RT06 Eval & N/A & 67.3 & 66.0 \\
\hline
\end{tabular}

Table 2: STT performance for single channel and beamformed array output using IEKF and JPDAF position estimates.

\section{Acknowledgements}

This work was sponsored by the European Union under the integrated project CHIL, Computers in the Human Interaction Loop, contract number 506909.

\section{References}

[1] Ulrich Klee, Tobias Gehrig, and John McDonough, "Kalman filters for time delay of arrival-based source localization," Journal of Advanced Signal Processing, Special Issue on Multi-Channel Speech Processing, to appear.

[2] M. S. Brandstein, J. E. Adcock, and H. F. Silverman, "A closed-form location estimator for use with room environment microphone arrays," IEEE Trans. Speech Audio Proc., vol. 5, no. 1, pp. 45-50, January 1997.

[3] Tobias Gehrig, Kai Nickel, Hazim Kemal Ekenel, Ulrich Klee, and John McDonough, "Kalman filters for audio-video source localization," in Proc. Workshop on Applications of Signal Processing to Audio and Acoustics, New Paltz, New York, 2005.

[4] G. Gennari and G. D. Hager, "Probabilistic data association methods in the visual tracking of groups," in Proc. CVPR, 2004, pp. 1063-1069.

[5] Dirk Bechler, Akustische Sprecherlokalisation mit Hilfe eines Mikrofonarrays, Ph.D. thesis, Universität Karlsruhe, Karlsruhe, Germany, 2006.

[6] S. Kay, Fundamentals of Statistical Signal Processing: Estimation Theory, Prentice-Hall, Englewood Cliffs, NJ, 1993.

[7] M. Omologo and P. Svaizer, "Acoustic event localization using a crosspower-spectrum phase based technique," in Proc. ICASSP, 1994, vol. II, pp. 273-6.

[8] J. Chen, J. Benesty, and Y. A. Huang, "Robust time delay estimation exploiting redundancy among multiple microphones," IEEE Trans. Speech Audio Proc., vol. 11, no. 6, pp. 549-57, November 2003.

[9] S. Haykin, Adaptive Filter Theory, Prentice Hall, New York, fourth edition, 2002.

[10] Y. Bar-Shalom and T. E. Fortmann, Tracking and Data Association, Academic Press, San Diego, 1988.

[11] T. Gehrig and J. McDonough, "Tracking multiple simultaneous speakers with probabilistic data association filters," in Proc. MLMI, 2006.

[12] Z. Zhang, "A flexible new technique for camera calibration," IEEE Trans. Pattern Analysis Machine Intel., vol. 22, pp. 1330-1334, 2000.

[13] C. Fügen, M. Wölfel, J. McDonough, S. Ikbal, F. Kraft, K. Laskowski, M. Ostendorf, S. Stüker, and K. Kumatani, "Advances in lecture recognition: The ISL RT-06s evaluation system," in Proc. Interspeech, submitted for publication, 2006. 